\title{
Testing an app-based intervention to improve insomnia in patients with epilepsy: A randomized controlled trial ${ }^{\text {2h }}$
}

\author{
Daniel Kwasi Ahorsu ${ }^{\mathrm{a}}$, Chung-Ying Lin ${ }^{\mathrm{a}}$, Vida Imani ${ }^{\mathrm{b}}$, Per Carlbring ${ }^{\mathrm{c}}$, Annette Nygårdh ${ }^{\mathrm{d}}$, Anders Broström ${ }^{\mathrm{d}, \mathrm{g}}$, \\ Kyra Hamilton ${ }^{\mathrm{e}}$, Amir H. Pakpour ${ }^{\mathrm{d}, \mathrm{f}, *}$
}

a Department of Rehabilitation Sciences, Faculty of Health E' Social Sciences, The Hong Kong Polytechnic University, Hung Hom, Hong Kong

b Pediatric Health Research Center, Tabriz University of Medical Sciences, Tabriz, Iran

c Department of Psychology, Stockholm University, Stockholm, Sweden

d Department of Nursing, School of Health and Welfare, Jönköping University, Jönköping, Sweden

e School of Applied Psychology, Menzies Health Institute Queensland, Griffith University, Brisbane, Queensland, Australia

${ }^{\mathrm{f}}$ Social Determinants of Health Research Center, Research Institute for Prevention of Non-Communicable Diseases, Qazvin University of Medical Sciences, Qazvin, Iran

${ }^{g}$ Department of Clinical Neurophysiology, University Hospital, Linköping, Sweden

\section{A R T I C L E I N F O}

\section{Article history:}

Received 2 April 2020

Revised 22 July 2020

Accepted 22 July 2020

Available online $\mathrm{xxxx}$

\section{Keywords:}

Epilepsy

Sleep

Insomnia

CBT

\begin{abstract}
A B S T R A C T
Purpose: Insomnia has adverse effects on people with epilepsy. We aimed to test a novel cognitive behavioral therapy for insomnia (CBT-I) app-based intervention on insomnia symptoms and social psychological factors in people with epilepsy and to examine the possible mechanisms among the factors.

Methods: Participants were recruited from neurology clinics in Iran and comprised individuals diagnosed with epilepsy and having moderate to severe insomnia. A two-arm randomized controlled trial design was used, consisting of a treatment group (CBT-I; $\mathrm{n}=160$ ) and control group (patient education; $\mathrm{n}=160$ ). Primary outcomes were self-reported sleep quality, insomnia severity, and sleep hygiene behavior and objective sleep characteristics measured by actigraphy. Secondary outcomes were attitude, perceived behavioral control, intention, action planning, coping planning, behavioral automaticity, self-monitoring, anxiety, depression, and quality of life (QoL). All outcomes were measured at baseline, and at one, three, and six months postintervention, except objective sleep, which was assessed at baseline, and one and six months postintervention. Data were analyzed using linear mixed models.

Results: Current findings showed that sleep quality, insomnia severity, sleep hygiene behavior, and sleep onset latency were significantly improved in the CBT-I group compared with the patient education group at all measurement points. Also, the CBT-I group had significantly improved anxiety, depression, and QoL compared with the patient education group. Mediation analyses showed that attitude, intention, coping planning, self-monitoring, and behavioral automaticity significantly mediated the effect of the intervention on sleep outcomes. Conclusion: Results support the use of the CBT-I app to improve sleep outcomes among people with epilepsy.
\end{abstract}

(c) 2020 Elsevier Inc. All rights reserved.

\section{Introduction}

Epilepsy is one of the most common neurological diseases globally, affecting around 50 million people worldwide [1]. Individuals with epilepsy often have other physical and mental health conditions [2]. For example, higher incidences of anxiety, depression, and sleep-wake disorders are commonly observed in people with epilepsy [2], all of which have been shown to be associated with insomnia [2].

\footnotetext{
it ClinicalTrials.gov NCT03683381.

* Corresponding author at: Social Determinants of Health Research Center, Research Institute for Prevention of Non-Communicable Diseases, Qazvin University of Medical Sciences, Shahid Bahounar BLV, Qazvin 3419759811, Iran.

E-mail address: Pakpour_Amir@yahoo.com (A.H. Pakpour).
}

Insomnia is characterized by difficulty initiating or maintaining sleep or early-morning awakening with an inability to return to sleep despite good opportunities for sleep [3]. Insomnia can have significant negative consequences on a person including disruptions to psychosocial functioning (e.g., depression, daytime dysfunction, reduced quality of life $(\mathrm{QoL})$ ) and occupational functioning (e.g., job absence, reduced ability to do tasks, lowered job satisfaction, poor decision-making), as well as an increased burden on society (e.g., increased health costs, reduced job productivity) [2-4]. Several treatment options have been used to manage insomnia in people with epilepsy; however, treatments are often dependent on the seizure type, the person's age, preexisting medical conditions, and potential side-effect profile [5]. Moreover, in Iran, the usual practice for improving sleep hygiene for those who have sleep difficulties is providing education through the provision of 